\title{
How can mobile SMS communication support and enhance a first year undergraduate learning environment?
}

\author{
Geraldine Jones*, Gabriele Edwards and Alan Reid \\ Department of Education, University of Bath, Bath, UK
}

(Received 14 January 2009; final version received 23 July 2009)

\begin{abstract}
In this paper we discuss a case study investigating how the academic and personal development of first year students on an undergraduate sports education degree can be supported and enhanced with mobile SMS (Short Message Service) communication. SMS-based technologies were introduced in response to students' particular needs (in transition to Higher Education) and characteristics (adept mobile communicators). Despite being unaccustomed to using their mobile phones for academic study, students willingly accepted SMS communication with their tutor via a texting management service. This communication was used in concert and integrated with a more traditional learning and teaching context (lectures and a virtual learning environment). Drawing on evidence from two student surveys, focus groups and a tutor's journal, we illustrate how mobile SMS communication has influenced the student learning experience. Taking a holistic view of the learning environment we use Laurillard's (2002) conversational framework (Laurillard, D. 2002. Rethinking university teaching: a framework for the effective use of learning technologies. 2nd edition. London: Routledge.) to analyse and discuss the role of texting in supporting student transition to higher education.
\end{abstract}

Keywords: SMS; mobile phones; transition; conversational framework; academic texting

\section{Introduction}

Supporting students at vulnerable points in their academic career has always presented challenges. When students make the transition to higher education (HE), they often need to accommodate and assimilate new modes of study given the advent of new academic demands or teaching arrangements that differ from their previous experiences in school or college. Meanwhile, the pressures on HE institutions to increase student numbers and employ more teaching staff on part time contracts can make it difficult for teaching staff to keep connected with their students. Thus supporting contemporary students through the transition to $\mathrm{HE}$ may require facilitating and maintaining connectivity between tutors and students, cognitively, physically and technologically.

The last point is important. The latest generation of undergraduates have grown up in a world of pervasive digital technology where widespread ownership of mobile devices has provided an infrastructure that these students rely on for building

*Corresponding author. Email: g.m.jones@bath.ac.uk 
extensive social communication networks (Reid and Reid 2005) in ways often unfamiliar to their tutors. Few studies have explored how to harness these infrastructures for study or transition purposes. Furthermore, little is known about how mobilemediated communication might complement more traditional learning and teaching contexts (e.g. lectures, seminars and more recently and increasingly, within virtual learning environments (VLEs)).

M-learning can be defined in many ways depending on the focus on the learner, technology, or learning context (Sharples, Taylor, and Vavoula 2007). Here we are concerned with the additional channels of communication afforded by the mobile phone that is a constant 'companion' of contemporary undergraduate students. Our interest is in how SMS (Short Message Service) can complement and enhance traditional HE education, while at the same time, address issues of disconnection between students and their tutors during periods of vulnerability, such as during the transition to HE.

In this paper we offer a case study that illuminates the role of mobile SMS, and issues associated with embedding this communication in an academic course of study. We believe this case to be of both intrinsic and instrumental value (Stake 2000) so we include a description of the case followed by an analysis using the conversational framework (Laurillard 2002), to investigate how SMS contributes to the overall learning environment.

\section{Transition to higher education}

There is now growing recognition that higher priority must be given to addressing the needs of students during their transition into HE (Kember 2001). First year students often report differences between the degree of support they received before entering $\mathrm{HE}$ and the support they receive in HE where they are expected to rely more heavily on their own efforts (Teese 2002). The first year experience has been recognised as especially important because this is when the majority of student departures occur, with Yorke (2002) noting that in the UK, around ten per cent of full-time HE students withdraw during or at the end of their first year. Bryson (1997) notes that the removal of familiar structures (e.g. family, rules, regulations and syllabus of a school) results in a difficult transition period for students, characterised by uncertainty. Placed in an academic environment in which self-direction and independence in learning are emphasised in contrast to more didactic approaches experienced in secondary education, many students feel isolated and insecure. Furthermore, little or no interaction or personal contact with tutors may lead to less confident students not succeeding in their first semester.

Tinto (2002) illuminates the socio-economic, cultural and institutional conditions which foster retention succinctly, including that students are more likely to persist in settings that provide academic, social and personal support. "Frequency and quality of contact with faculty, staff, and other students is an important independent [sic] predictor of student persistence" (2). Kift and Nelson (2005) review institutional practices that support learners, and posit that for successful transition, HE should inculcate a sense of belonging via involvement, engagement and connectedness.

A recent study by Jones (2008) on the effects of out-of-class support on student satisfaction and motivation highlights that such contact "demonstrates a responsiveness to students' needs; communicates caring; validates students' worth [...] and helps students manage and cope with stressful situations..." (375). Social presence, 
intimacy and immediacy have been researched by Short, Williams, and Christie (1976), for example, while Steffey (2001) summarises that social presence was found to be consistently associated with learner satisfaction, and assumed to influence motivation (in Rau, Gao, and $\mathrm{Wu} 2008$ ).

Ferrante et al.'s (2008) study provides rich representative metaphors exemplifying students' experience of “connection and disconnection" (112). These authors urge higher education institutions to build connections, and demonstrate that out of classroom communication between students and teachers is vital.

\section{SMS in a social context}

Mobile phones can now be considered as pervading the UK student population. Ofcom (2007) reports that in 2006, 96\% of the UK population in the 15-24 age group personally used a mobile phone. More provocatively, the Horizon Report (Johnson, Levine, and Smith 2007) predicts that "the capabilities of mobile phones are increasing rapidly, and the time is approaching when these little devices will be as much a part of education as a bookbag." As young undergraduate students enter HE, bringing with them personal mobile devices and expertise in mobile communication, arguably there will be increasing demands for more mobile communication on campus. The social contexts surrounding students' mobile communication practices may help us understand these demands. The Mobile Life (2008) survey shows that $63-66 \%$ of the US and British population use their mobile phones for keeping in touch with family and friends, and more specifically, $50 \%$ of British young people send more than six text messages per day. Mitchell and Doherty (2003) posit that "SMS is more than just a passing fad; it is a communication medium integral to young people's lives" (11). Furthermore, complex systems of social etiquette are brought into play by young people to manage close relationships through this medium (Harper and Hamill 2005). However, according to some researchers (Thurlow 2003; Kim et al. 2007), it seems that SMS is more likely to add to and blend with face-to-face communication rather than replace it. Thus Reid and Reid's (2005) research cautions against generalisations suggesting uniformity in young peoples' preferences or approaches to SMS communication for social purposes.

The social motivation for texting is clearly significant for educators seeking to adopt this technology as part of a learning environment; however, the form as well as the function of the messages themselves is also of potential interest. Text messages typically exhibit idiosyncratic forms, driven in part by a restrictive length (160 characters), and the speed and cost of messaging (Thurlow 2003). Against this backdrop of 'social' mobile communication we now consider what progress has been made with integrating SMS into formal educational settings, specifically HE.

\section{SMS communication for classroom work and administration}

Stone, Biggs, and Smith's (2002) experiments reveal the effectiveness of SMS in gaining attention compared to email. For time-critical administrative communication (e.g. delayed start to a lecture), Griffiths and Hmer (2004) conclude SMS is more reliable than email in reaching the recipient. Nix, Russell, and Keegan (2006) suggest that using mobile phones and SMS for academic administration had a positive impact on drop out rate and supporting 'at risk' students. Naismith (2007) advises that effective administrative messages should be "time-sensitive, relevant, unambiguous, selective 
and trustworthy" (166). Traxler and Riordan (2003) used an SMS system to distribute feedback on assignments and revision tips to undergraduate students. These studies showed students were generally supportive of SMS communication as long as no cost was incurred. The MeLAS project (Brett 2008) extends this empirical research through a large-scale implementation of SMS communication across a whole HEI. The focus was on the pedagogic potential of SMS, including quizzes and conferences, indicating students were generally less supportive of texting connected with their course content than for administration.

A number of smaller scale studies also explore the characteristics of SMS for pedagogical purposes, some taking SMS into the HE classroom reporting increased interactivity for students, feedback for tutors (Markett et al. 2006), and more peer learning opportunities (Cheung 2004). However, a proportion of students (33\%) experienced cognitive overload when attending to a lecturer while simultaneously composing an SMS question (Markett et al. 2006).

Using an asynchronous approach, Thornton and Houser (2004) show pedagogical benefits when small content items are sent to student phones as compared to email or posting on a website. Levy and Kennedy (2005) found that messages closely integrated with content on a modern languages course promoted an "interconnectedness between in-class and out-of-class activity" (81). Ng'ambi (2005) implemented a dynamic FAQ system incorporating SMS where anonymity was key, enabling a more inclusive learning environment. Overall these studies show student willingness to use SMS outside a social context and highlight the potential for SMS to support learning in HE.

\section{Perceived advantages of mobile communication for transition}

Pastoral support is the focus of Horstmanshof's (2004) work and Harley et al.'s (2007) focus on transitioning. Both studies demonstrate student willingness to accept their tutors as participants in informal SMS communication about their courses of study. Horstmanshof used her own mobile phone and encouraged students to make contact, while Harley et al. planned messages to be sent via a computer-based SMS system at perceived vulnerable times. Harley et al. exploited the system of communication already operating amongst students, and found that "it gave them a sense of belonging to the university" (236). Similarly, Rau, Gao, and Wu $(2008,11)$ found students reported "feeling cared for, and felt bonded strongly with the instructor and classroom activities", and, importantly, reported significant differences in motivational items (op cit.). Stone (2004) sought to improve retention rates through a 'mobile scaffold' of administrative messages. While there are legitimate concerns about the misuse and abuse of these support mechanisms (see below), these studies highlight the significance of immediacy and intimacy with respect to SMS communication for transition.

\section{Perceived disadvantages of mobile communication for transition}

The introduction of mobile communication into an academic environment is not a panacea - it can bring problems as well as solutions. Sharples (2007), for example, calls for caution from the students' perspective, suggesting that young people may see the use of SMS for formal learning as an attempt to colonise and intrude on social spaces. Criticisms about encouraging superficial communication and thinking (Mason and Rennie 2008) have also been mounted against SMS use in academic contexts. 
Rheingold (2004) reports concerns about disruption of formal learning contexts with the introduction of student-owned mobile devices and the social practices surrounding their use. There is potential for the classroom or lecture theatre to become an unwelcome virtual extension of social space. In Markett et al.'s (2006) study, students were challenged by SMS communication interleaved with face-to-face interaction. This blurring of boundaries has been found to provide unwelcome distractions, and could thereby potentially make the transition to HE more challenging. Hortsmanshof (2004) discusses two concerns related to students in transition; firstly that of nurturing a dependence on support structures which could hamper the development of more self-regulatory strategies, and secondly, the potential burdens placed on tutoring staff in managing an additional communication channel.

\section{Case study}

\section{Context}

Our focus is on a first year undergraduate unit, 'Exploring Effective Learning', at the University of Bath. The aims of the unit are to identify effective approaches to learning, and develop student understanding of the learning process in the context of study in HE. The unit is well established, where teaching consists of two hours contact per week, with an expectation of students mirroring this in regular independent study. Coach Education and Sports Development students experience particular challenges with transition as there are extra demands on their time, due to their involvement in sport. Most students are resident on campus, and they also have easy access to the VLE.

Unit reports from previous cohorts suggest that students have difficulty in sustained engagement with independent study as evidenced by the tutor's observations of poor responses to set tasks, declining attendance at lectures over the semester, and superficial use of personal learning journals to support assignment tasks. Although the learning environment had potential for rich dialogue between students and tutor, this was rarely realised. This impoverished learning environment with respect to dialogue and interaction may be viewed in terms of weakened transactions in Laurillard's (2002) conversational framework (see Figure 1).

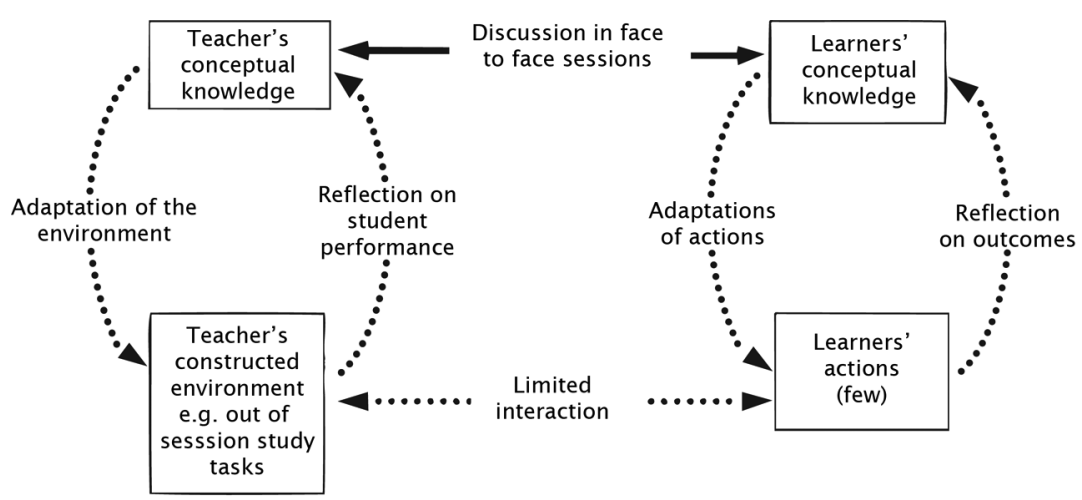

..... Weakened or non existent transactions

Figure 1. Student learning environment viewed in terms of weak or non-existent transactions within the conversational framework (Laurillard 2002). 


\section{Aims}

By structuring inter-session study time using group learning activities (JISC 2005) we aimed to establish networked opportunities for learning that more effectively support students on this unit and motivate student participation. In addition we sought to harness the communication cultures and skills of these contemporary students, using mobile phones and SMS to promote greater connectivity between tutor and student, and student and course content.

\section{Preliminary investigation}

We surveyed the new students $(n=56)$, prior to arrival on campus about the extent of mobile phone ownership, the capability of their devices, and the range of contracts or licences, student cultures and skills surrounding mobile use, alongside the students' willingness to adopt mobiles as tools to support their learning.

Students responded that they were almost always available for communication via their mobiles. They reported checking for messages frequently (e.g. every $20 \mathrm{~min}$, every hour, several times a day) and always responding to the arrival tone. The majority of students owned high specification mobiles. For example $91 \%$ had camera phones, $87 \%$ had phones with web-browsing capability, $89 \%$ had Bluetooth enabled phones and all had phones with built-in calendar functionality. Surprisingly few seemed to use anything more than basic capabilities of SMS and voice calls, a finding that runs counter to the image portrayed through the popular conception of the new generation of students as 'digital natives' (Prensky 2001). Figure 2 shows the percentage of students who rarely or never used their mobile phones to carry out range of possible tasks.

The vast majority of students said they would welcome SMS contact with tutors. However the contact they welcomed most was receiving questions, rather than asking questions or generally keeping in touch with their tutors (Figure 3). All were willing to receive SMS connected with their course (particularly reminders about deadlines, Figure 3) at least once or twice a week. Most (97\%) were willing to use some of their

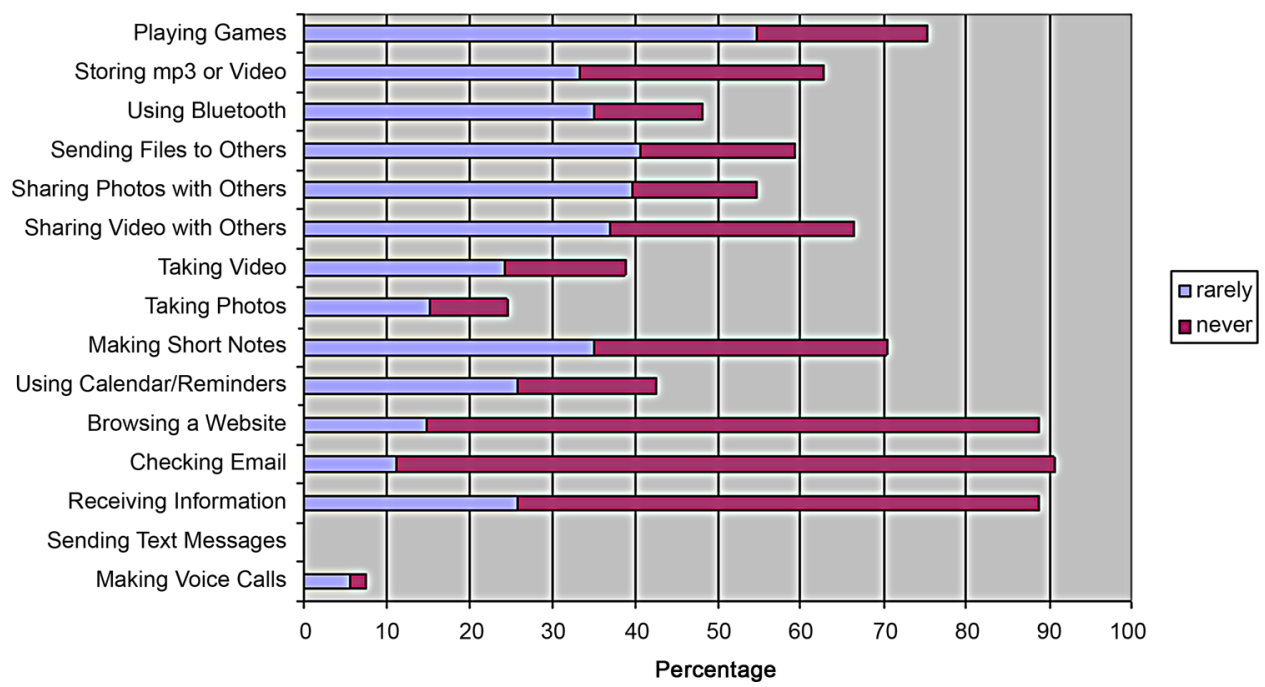

Figure 2. 'Redundant functionality' of student-owned mobile phones. 


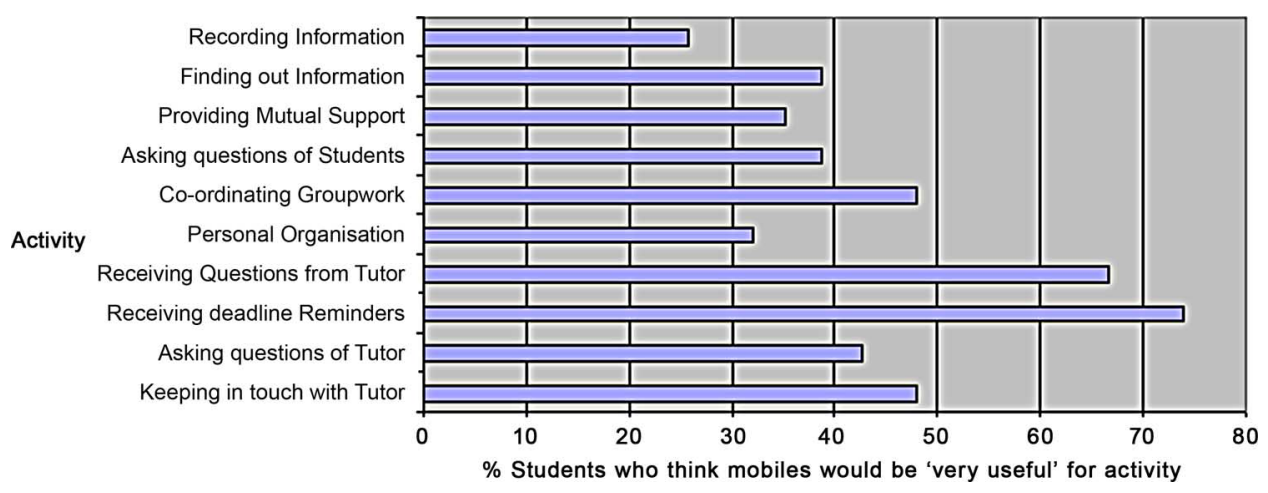

Figure 3. Student views about the potential for using mobile phones to support learning.

own texts for study purposes. Students were happy to allow their mobile numbers to be stored securely by their department.

\section{Implementation}

Our literature review and preliminary survey provided us with guidance for the design of an SMS-enabled learning environment aiming to facilitate greater connectivity between students and their tutor, thereby potentially helping to support transition. For example, Levy and Kennedy's (2005) work gave advice for promoting greater interconnectedness between in- and out-of-class activity by closely linking texts to course content. In order to minimise any possible disruption to classroom contexts and student social communication networks we adopted an asynchronous approach where tutor talk moved from the face-to-face context into the mobile environment and back to face-to-face, a pattern familiar to social texters (Kim et al. 2007). Guidance from our survey regarding the students' views of acceptable use of text messaging for learning, especially volume, timing and purpose of the texts also influenced our design.

The tutor talk normally located at the end of a formal session (directives, hints, questions and advice that signpost private study), was moved to out-of-session time and delivered via SMS scheduled at strategic times during the week.

Following a face-to-face session the tutor sent out text messages to students highlighting a relevant task located in the VLE. Typically these tasks were designed to elicit individual responses that were shared with others via the Moodle activity tools (e.g. choice or wiki). The multiple choice activity prompted categorisation of key statements and the wiki activity guided each student to contribute experiences to a single structured webpage. Some tasks took place in a forum, however discussion was limited. Interactions in Moodle were largely student-student and student-content. The tutor was minimally involved in the online activities, her communication with the students was predominantly face-to-face or via SMS (see Figure 5 for examples) and to a lesser extent via email generated by the VLE. Prior to a face-to-face session the tutor sent further text messages to students detailing preparatory activities. Students were requested to respond via SMS. These responses (see Figure 6 for examples) and the products of student activity in the VLE were collected and incorporated into the next face-to-face session where feedback was given. These materials also provided sparks for further face-to-face discussion. 


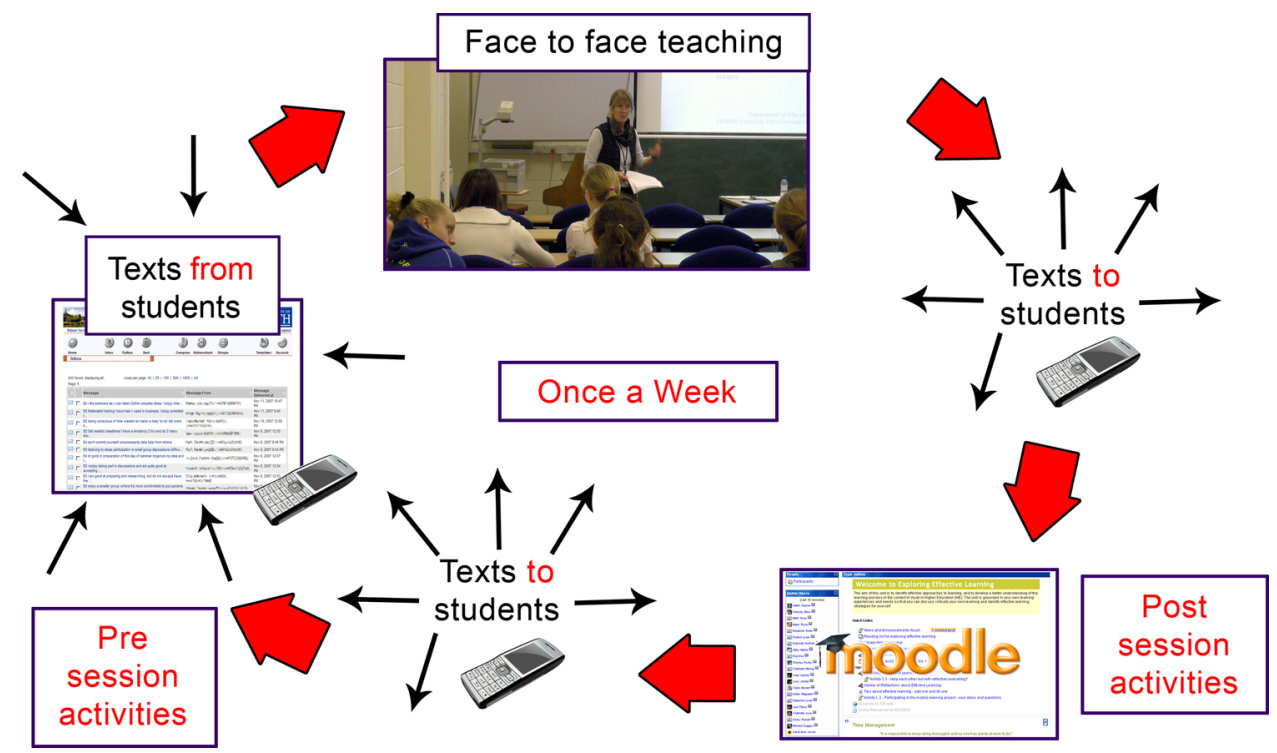

Figure 4. SMS enabled learning environment.

To achieve efficient management and distribution of messages to students, the tutor used a texting management service (Edutxt). The 'email-like' web-based user interface enabled easy composition, scheduling and sending of personalised SMS using a 'mail merge' option. Messages going out to students were carefully timed to avoid unsocial hours ( $5 \mathrm{pm}-11 \mathrm{am}$, as advised by the students). In order to maximise the chances of students being able to act on messages and to engender a routine of outof-class study, messages were scheduled to arrive at similar points in the week when the students had no other lectures or timetabled activity. The tutor obtained immediate feedback regarding successful delivery of the messages via the Edutxt interface. When appropriate, the tutor was able to export (as a spreadsheet) messages received from students to share (anonymously) with the group. Students had no access to each others' SMS apart from this route. Figure 4 summarises the implementation of this SMS enabled learning environment.

Hi Tom. A reminder 2 lk at $\&$ comment on the reading improvement video-Moodle [Act 4.1] Pls txt in wht $u$ hve learned from the video b4 session. Gabriele

Hi Laura. A reminder [if
needed] to text in your
definition of
metacognition before
2pm today. Start your
text with ED. C u
2moro. Gabriele
needed] to text in your definition of 2pm today. Start your 2moro. Gabriele

Hi lan, jst a reminder to think of a recent lecture \& txt in what the lecturer did to help/hinder yr learning in the lecture. By 9am 2moro pls. Gabriele
Hi Joanna, A remnder 2 complete follow-up acts $[5.4,5.5]$ good prep for assgnmnt, [5.6] ur action plns. Lecture style videos now on Moodle. Gabriele

Figure 5. Examples of text messages sent to students.

i have learnt how The sq3r strategy could help me 2 read aswel as actually takin in the info \& learning at the same time e.g. Taking notes at the same time awareness + understandin of $1 \mathrm{~s}$ own 4 t proceses esp regarded as havin a role in directin those processes
The lecturer is willing to answer any questions that anyone has, and uses specific language that students will understand

Figure 6. Examples of text messages received from students. 


\section{Summary of findings}

A student survey, access statistics from the VLE and the texting management service, student focus groups, and the tutor's journal all contributed data to our findings. Overall, students highly valued the text messages as part of this unit, only two chose to opt out. A questionnaire was administered at the end of the unit (50 questions, both qualitative and quantitative). The response rate was $88 \%(\mathrm{n}=81)$. All respondents answered the quantitative questions however fewer answered the qualitative questions. When asked about how well the face-to-face teaching, the VLE, and the texts had worked together, 74\% $(\mathrm{n}=71)$ thought they had worked well or very well together. Students thought texting had added to their learning on the unit predominantly through convenience/accessibility/gaining attention $(36 \%, \mathrm{n}=59)$ or through prompting them to take action $(32 \%, \mathrm{n}=59)$. Generally students took positive actions with the texts e.g. acting on it, saving it; however $11 \%(n=71)$ chose to ignore or delete messages about the unit. The focus groups discussed how they responded to the texts. Examples included: Student S: receiving a text while playing computer games - switches tasks; H: keeps it on her phone for later; A: likes to see the whole weeks' worth of tasks yet regards texting as disruptive of her personal organisation.

The VLE and texting management service statistics suggested that there was little activity in the VLE prior to students receiving texts from their tutor. Subsequently they successfully and consistently guided students to the intersession learning activities in the VLE, in some cases just a few minutes after receiving their message (Figure 7).

Students welcomed texts as reminders about study tasks, deadlines, administrative changes, etc., and regarded them as an effective aid to time management $(62 \%, \mathrm{n}=$ 71 ), especially in the busy first few weeks of term. They felt that the texts were better than email at gaining their attention and commented (focus group) that their email had been colonised by Facebook. They saw the texts as clarifying instructions that could easily be missed at the end of face-to-face sessions. Students liked the personal nature of the texts and suggested that they could be appropriate for personal tutoring. When asked about the difference between receiving SMS from their tutor and setting their own reminders or alarms on their phones, students thought the messages sent from their tutors were more effective acting as an extrinsic motivator in helping them to study. However, on a few occasions, texts arrived at inconvenient times. Few students

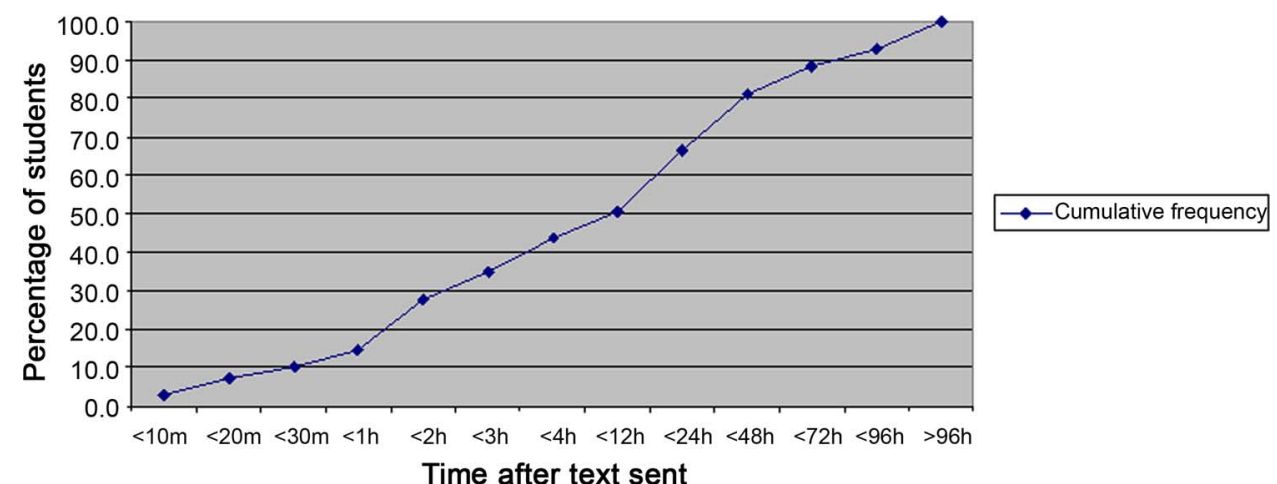

Figure 7. The relationship between the time the text messages were sent out and students' arrival in the virtual learning environments (VLE). 
How do you feel when you receive a text from the course?

Which of the following statements do you identify with?

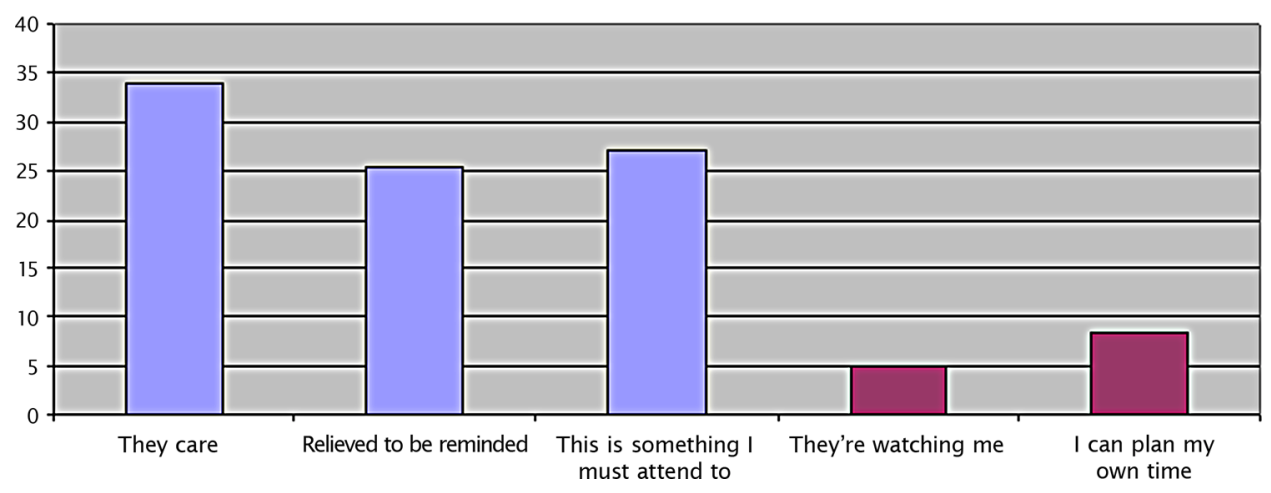

Figure 8. Percentage of students identifying with particular feelings when receiving studyrelated SMS.

had strong general feelings about receiving texts (see Figure 8) and only a minority $(13 \%, n=71)$ expressed negative reactions to them associated with message redundancy (feeling 'nagged') or inappropriate timing (feeling minor irritation).

Focus group discussions revealed that students saw texting back comments in response to learning activities as more problematic than receiving texts from their tutor. Some found 160 characters to be inhibiting while others saw it as a positive challenge. Sometimes composing texts that required academic vocabulary, new abbreviations or predictive text proved difficult. Other inhibitors included no course credit $(24 \%, n=55)$ or were associated with doubts about task efficacy. In a few cases, lack of signal on campus was a problem and a few students found themselves locked into poor network coverage.

Although managing and manipulating large numbers of texts was unproblematic for staff, there still remained challenges associated with choosing appropriate purposes for SMS, composing meaningful 160-character messages and mastering texting language. Students commented that occasionally the tutor's texts appeared unnatural or that they were difficult to read, for example when she used unconventional abbreviations like 'yr' instead of 'ur' or when she created her own abbreviations of academic words.

Reading large numbers of text messages authored by peers was challenging for students, particularly when they contained many abbreviations (described by students as 'gangsta txts'). This was ameliorated in part by presenting collective responses back to the group as a 'word cloud' (a visual representation of the words used, with those used more frequently displayed in a larger font). However, the tutor noted a positive effect in terms of interest and discussion when group responses (Appendix 1) from the inter-session learning activities were brought into sessions. She regarded the texts received from students as a valuable way of surveying opinion and engaging the students prior to a face-to-face session.

Overall the tutor's view was that SMS had contributed to increased and sustained student engagement with the unit (as evidenced by better attendance throughout the semester), increased participation in learning activities, and a better quality of work from the lower-achieving students as compared to previous years (Figure 9). We make 


\section{Assignment Results}

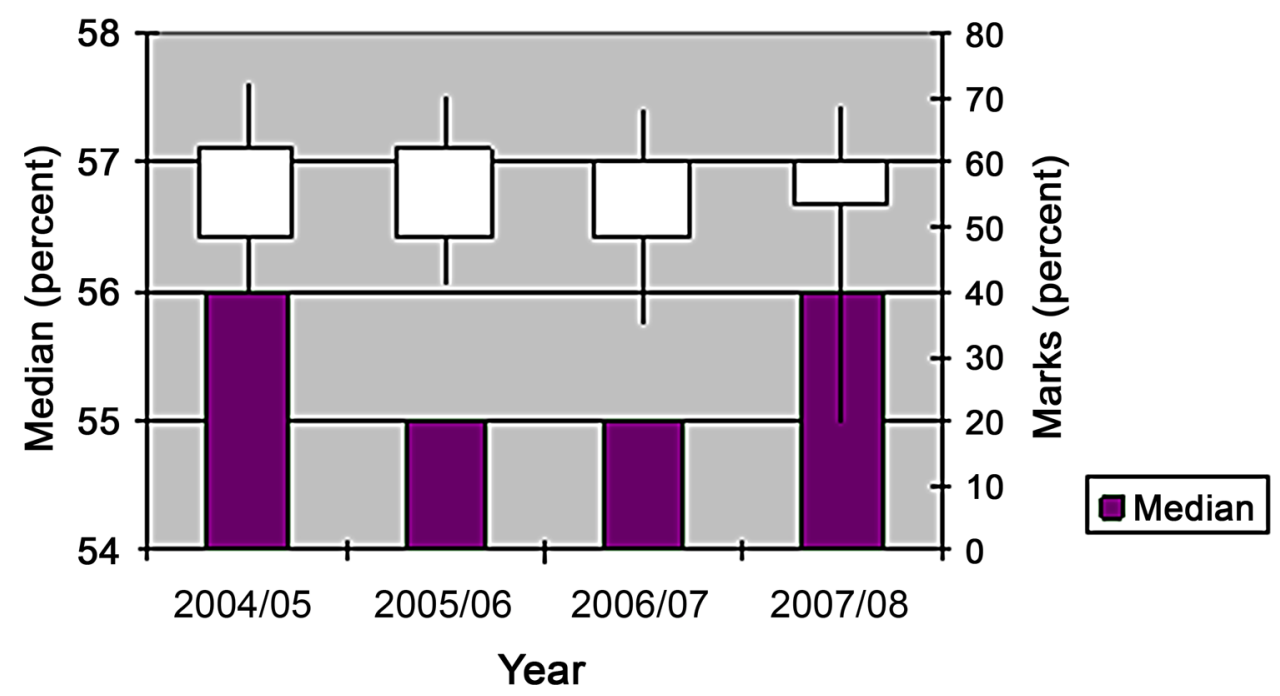

Figure 9. Comparison of median, upper and lower quartile assignment scores .

no claims about a causal link here as many factors could be at play. We merely note the assignments' results as an indicator of quality of work.

\section{Discussion}

The attributes (student negotiated, regular, personal, content related) of our particular regime of out-of-class SMS communication can, according to the literature, be linked to successful strategies for supporting student transition to HE. Although this communication could have been facilitated by other means, for example by email via the VLE, we argue that SMS offers distinct advantages when used in this role. Students by their own admission were more 'tuned into' SMS communication than email and therefore it was more effective in gaining attention. Also text messages appear more personal as they are delivered to a personal mobile and address the recipient by name (e.g. Hi Kerry!). Face-to-face, calling students by name is a powerful instructional immediacy strategy. This and other manifestations of immediacy have been researched; for example, Allen, Witt, and Wheeless (2006) have linked the positive impact of teacher immediacy behaviours on student motivation. Both Horstmanshof (2004) and Harley et al. (2007) report on the motivational value of personal text messages in conjunction with student support.

Texting in our case study was closely integrated with learning activities that crossed between face-to-face and the VLE. To try to understand how the texting was impacting on the learning environment as a whole we turn to Laurillard's (2007) framework as an analytical tool for determining what technology brings to a learning process, asking, "how much of the framework does the technology support?"

From Laurillard's framework we can see that the personal text messages received by students prompted out-of-session study actions, for example participation in group activities in the VLE or sending a text response to the tutor. Arguably, and according 
to the framework, outcomes of these learner actions would have provided richer opportunities for reflection compared to previous cohorts (Figure 1), both on the part of the tutor and the students. Strengthening the reflective pathways enabled the tutor to adapt subsequent study tasks to meet evolving student needs and gave students more opportunity to learn from each other and make more informed decisions about future study actions.

For Laurillard, each of the transactions in the conversational framework plays its part in motivating other transactions, creating a continual iterative flow: "good learners may take themselves around these iterative loops...but poor learners need the teacher to construct their learning environment in such a way that they can scarcely avoid being active learners" (162). In our context, we take 'good' to mean 'more experienced' learners, and 'poor' to mean 'new' learners or those 'in transition'. According to our findings the texts were providing additional value as personal and motivating directives for out-of-session study time. We suggest this was instrumental in enabling the cycle of activities around the conversational framework to be maintained. Indeed there is evidence that overall there was regular engagement with out-of-session activities over the course of the unit and students themselves reported that they viewed the texts as prompts to work and that they acted to support their time management.

Although this study included no direct measures of the success or otherwise of transition, it is our contention that by using SMS to (1) increase the opportunity for tutor student interaction, (2) adopt a highly personal mode of communication, and (3) stimulate regular out-of-class activity, our approach aligns well with strategies for supporting a successful transition.

While Laurillard's (2007) framework provides a useful generalisation, it is less helpful in highlighting differences between individual student experiences or indeed changes in the same individual's experience over time. There were indications from our study that the student experience of SMS was not uniform across the group. A minority saw the texts as disruptive of their own organisational strategies. We could speculate that these students might have already progressed some way through transition (as evidenced by better developed organisational skills) and therefore needed less support. Our study also showed differences in how students managed the texts on their phones and how they felt when they received texts from their tutor. This variation highlights that some students may have benefited more than others with respect to support for transition and participation in an enriched learning environment. Further research is needed to investigate the nature of this variation.

Surprisingly, both tutors and students were challenged at times by the language of text and the short message length. Students even found each other's academic texts difficult to interpret. This prompted us to question popular assumptions and generalisations about young people's familiarity and comfort with new technologies. Seemingly not all skills acquired using new technologies for social purposes transfer well to an academic context.

Returning to Laurillard's framework we see that problems associated with managing the texting language could potentially reduce the efficacy of the whole learning environment. If tutor texts were regularly being misinterpreted or if they proved entirely intractable to students, then SMS communication would be less efficient in leading students to out-of-class activities. However, evidence from our case study showed that texts were effective and timely in this respect. Some students were reticent to return responses to activities by text. Arguably, this had a lesser impact on 


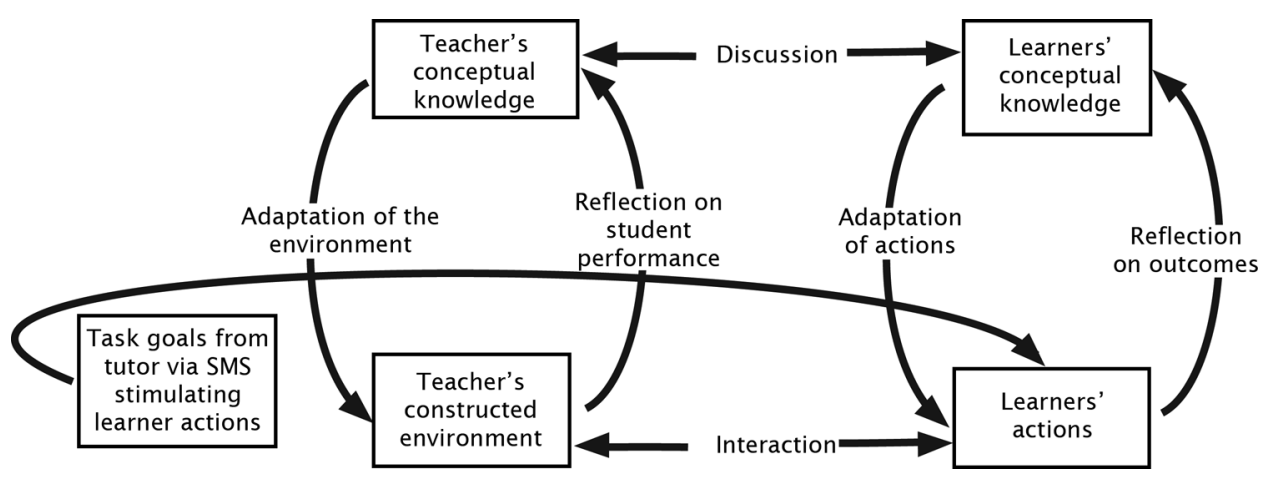

Figure 10. Using the Conversational Framework to locate the role of SMS communication.

the learning environment (Figure 10) as this was not the only route by which students could actively engage with the content.

To some extent we share Horstmanshof's (2004) concerns regarding possible consequences of dependency on text reminders, thus mitigating against developing more independent approaches to study. However, we saw the SMS communication as an additional means of supporting students in the early stages of transition only. As good study habits develop, students can choose to opt out.

Our study offers no evidence to support concerns voiced by others (Sharples 2007) that students see texting for academic purposes as an intrusion into their social space. Reasons for this could be connected with how we introduced the SMS communication, in negotiation with students. Also, it could be important that we aligned our academic SMS with existing social SMS protocols (Kim et al. 2007). In addition, each message carried administrative content, course-related information and, importantly, the tutor's voice, a blend which might have been significant in making them seem more acceptable.

\section{Conclusion}

Overall, it is our contention that the advantages of using SMS communication for supporting the transition to HE outweigh the disadvantages. We have argued that the highly personal nature of texting as a communication medium can represent a powerful tool for enhancing traditional teacher immediacy strategy and thereby contribute to narrowing the psychological space between tutor and students. Indeed in constructively well aligned learning environments Biggs (2003) suggests that the role of the teacher is as broker between the student and the learning environment. SMS communication in our study can be considered as making an important contribution to tutor brokering activity by helping to motivate participation in appropriate activities.

In addition, we have shown how the simple text message can play a small but key role in driving the learning process forward by catalysing critical communication pathways outlined in Laurillard's (2007) model.

Clearly, there appears to be some variation in how individual students react to texting in an academic environment. However, we contend that if the most vulnerable students benefit from an SMS-supported transition to HE and other students are not adversely affected, then mobile communication is a worthwhile addition to a first year undergraduate learning environment. 
The advent of digital mobile technologies and a growing interest in their deployment for m-learning has enabled us to think differently about communication for learning, not solely taking place in the fixed locations of lecture theatres and seminars rooms but as pervading HE. The extensive functionality of today's highly flexible personal mobile communication devices can be seductive, causing us to lose sight of the task in hand: that of providing effective learning experiences for our students. Through our case study we illustrate the importance of keeping grounded in the learning process and students' lifeworlds rather than the technology.

\section{Acknowledgements}

This paper was first presented at the Networked Learning Conference 2008.

\section{References}

Allen, M., P. Witt, and L. Wheeless. 2006. The role of teacher immediacy as a motivational factor in student learning: Using meta-analysis to test a causal model. Communication Education 55, no. 1: 21-31.

Biggs, J. 2003. Teaching for quality learning at university. 2nd edition. Buckingham: Open University Press.

Brett, P. 2008. The large scale implementation of SMS for learning and teaching. Paper presented at Third International Blended Learning Conference, June 19, in University of Hertfordshire, De Havilland campus, Hatfield, UK.

Bryson, J.R. 1997. Breaking through the A level effect: A first-year tutorial in student selfreflection. Journal of Geography in Higher Education 21, no. 2: 163-9.

Cheung, S. 2004. Fun and games with mobile phones: SMS messaging in microeconomics experiments. In Beyond the comfort zone: Proceedings of the 21st ASCILITE conference, ed. R. Atkinson, C. McBeath, D. Jonas-Dwyer, and R. Phillips, 180-3. Perth, Western Australia. http://www.ascilite.org.au/conferences/perth04/procs/cheung.html.

Ferrante, K., K.M. Olson, T. Castor, M. Hoeft, J.R. Johnson, and R.A. Meyers. 2008. Students' metaphors as descriptors of effective and ineffective learning experience. Practice and Evidence of Scholarship of Teaching and Learning in Higher Education 3, no. 2: $103-28$.

Griffiths, L., and A. Hmer. 2004. U R L8 4 ur exam : )—students' opinions towards receiving timely information via SMS. Paper presented at the 11th International Conference of the Association for Learning Technology, September 14-16, in Exeter, UK. http:// www.alt.ac.uk/altc2004/timetable/files/133/alt-c-2004-v1-LGAH\%20.ppt.

Harley, D., S. Winn, S. Pemberton, and P. Wilcox. 2007. Using texting to support students' transition to university. Innovations in Education and Teaching International 44, no. 3: $229-41$.

Harper, R., and L. Hamill. 2005. Kids will be kids: The role of mobiles in teenage life. In Mobile world: Past, present and future, ed. L. Hamill and A. Lasen, 61-74. Berlin: Springer-Verlag.

Horstmanshof, L. 2004. Using SMS as a way of providing connection and community for first year students. In Beyond the comfort zone: Proceedings of the $21^{\text {st }}$ ASCILITE Conference, ed. R. Atkinson, C. McBeath, D. Jonas-Dwyer, and R. Phillips, 423-7. Perth, Western Australia. http://www.ascilite.org.au/conferences/perth04/procs/horstmanshof.html.

JISC. 2005. Innovative practice with E-learning. Bristol: HEFCE.

Johnson, L.F., A. Levine and R.S. Smith. 2007. Horizon report. Austin, TX: The New Media Consortium. http://www.nmc.org/pdf/2007_Horizon_Report.pdf.

Jones, A.C. 2008. The effects of out-of-class support on student satisfaction and motivation to learn. Communication Education 57, no. 3: 373-88.

Kember, D. 2001. Beliefs about knowledge and the process of teaching and learning as a factor in adjusting to study in higher education. Studies in Higher Education 26, no. 2: $205-21$. 
Kift, S., and K. Nelson, 2005. Beyond curriculum reform: Embedding the transition experience. Paper presented at HERDSA 2005 Conference, July 3-6, University of Sydney, Sydney.

Kim, H., G.J. Kim, H.W. Park, and R.E. Rice. 2007. Configurations of relationships in different media: FtF, email, instant messenger, mobile phone, and SMS. Journal of ComputerMediated Communication 12, no. 4. http://jcmc.indiana.edu/vol12/issue4/kim.html.

Laurillard, D. 2002. Rethinking university teaching: A framework for the effective use of learning technologies. 2nd edition. London: Routledge.

Laurillard, D. 2007. Pedagogical forms for mobile learning: Framing research questions. In Mobile Learning: Towards a research agenda, ed. Norbert Pachler, 153-75. London: WLE Centre IoE.

Levy, M., and C. Kennedy. 2005. Learning Italian via mobile SMS. In Mobile learning: A handbook for educators and trainers, ed. A. Kukulska-Hulme and J. Traxler, 76-83. Oxford: Routledge.

Markett, C., I. Arnedillo Sanchez, S.B. Weber, and B. Tangney. 2006. Using short message service to encourage interactivity in the classroom. Computers \& Education 46, no 3: 280-93.

Mason, R., and F. Rennie. 2008. E-learning and social networking handbook: Resources for higher education. Oxford: Routledge.

Mitchell, A., and M. Doherty. 2003. M-learning support for disadvantaged young adults. Ultralab. http://www.m-learning.org/archive/docs/Cal03\%20paper\%20Ultralab\%20Apr\% 2003.pdf.

Mobile Life Report. 2008. The connected world. LSE: London. http://www.mobilelife2008. co.uk/Mobile_Life_2008.pdf.

Naismith, N. 2007. Using text messaging to support administrative communication in higher education. Active Learning in Higher Education 8, no. 2: 155-71.

Ng'ambi, D. 2005. Mobile Dynamic Frequently Asked Questions (DFAQ) for student learning and support. Paper presented at MLEARN 2005, October 25-28, in Cape Town, South Africa.

Nix, J., J. Russell, and D. Keegan. 2006. Mobile learning/SMS academic administration kit. Paper presented at the EDEN Take Learning Mobile conference, September, IADT, in Dublin.

Ofcom 2007. The Consumer Experience. Research Report. http://www.ofcom.org.uk/ research/tce/ce07/research07.pdf.

Prensky, M. 2001. Digital natives, digital immigrants. On the Horizon. NCB UP 9, no. 5: 1-6.

Rau, P.-L. P., Q. Gao, and L.-M. Wu. 2008. Using mobile communication technology in high school education: Motivation, pressure and learning performance. Computers \& Education 50, no. 1: 1-22.

Reid, D.J., and F.J.M. Reid. 2005. Textmates and text circles: Insights into the social ecology of SMS text messaging. In Mobile world: Past, present and future, ed. L. Hamill and A. Lasen, 105-18. Berlin: Springer-Verlag.

Rheingold, H. 2004. M-Learning 4 generation txt? The Feature Archives. http://www.thefeaturearchives.com/101157.html.

Sharples, M., ed. 2007. Big issues in mobile learning: Report of a workshop by the Kaleidoscope Network of Excellence Mobile Learning Initiative. Nottingham, UK: University of Nottingham, Learning Sciences Research Institute.

Sharples, M., J. Taylor, and G. Vavoula. 2007. Learning for the mobile age. In The Sage handbook of e-learning research, ed. C. Haythornthwaite and R. Andrews, 221-47. London: Sage.

Short, J., E. Williams, and B. Christie. 1976. The social psychology of telecommunications. London: Wiley.

Stake, R. E. 2000. Case studies. In Handbook of qualitative research. 2nd edition, ed. N.K. Denzin and Y.S. Lincoln, 237-47. Thousand Oaks, CA: Sage.

Steffey, C. 2001. The effects of visual and verbal cues in multi-media instruction. PhD diss., Virginia Polytechnic Institute.

Stone, A. 2004. Blended learning, mobility and retention: Supporting first-year university students with appropriate technology. Paper presented at MLEARN 2004: Mobile Learning anytime everywhere, July 5-6, in Rome, Italy. 
Stone, A., J. Biggs, and C. Smith. 2002. SMS and interactivity and its implications on effective uses of mobile technologies in education. In Proceedings of the IEEE international workshop on wireless and mobile technologies in education, ed. M. Milrad, U. Hoppe, and Kinshuk, 147-51, Växjö, Sweden.

Teese, R. 2002. Bridging the gaps between secondary and tertiary education. Paper presented at the Mind the Gap Conference, September 23, University of Melbourne, Australia.

Thornton, P., and C. Houser. 2004. Using mobile phones in education. In Proceedings of the 2nd IEEE workshop on wireless and mobile technologies in education, ed. J. Roschelle, T. Chan, Kinshuk, and S.J.H. Yand, 3-10, Taoyuan, Taiwan.

Thurlow, C. 2003. Generation txt? The socio-linguistics of young people's text-messaging. Discourse Analysis Online 1, no. 1: http://extra.shu.ac.uk/daol/articles/v1/n1/a3/thurlow 2002003-t.html.

Tinto, V. 2002. Taking student retention seriously: Rethinking the first year of college. Speech presented at the annual meeting of the American Association of Collegiate Registrars and Admission Officers, April 15, in Minneapolis, Minnesota.

Traxler, J., and B. Riordan. 2003. Evaluating the effectiveness of retention strategies using SMS, WAP and WWW student support. Proceedings of $4^{\text {th }}$ annual conference, 54-5. Galway, Ireland: LTSN Centre for Information and Computer Science.

Yorke, M. 2002. Formative assessment - the key to a richer learning experience in semester 1. Exchange 1: 12-3. 


\section{Appendix 1. Text messages (verbatim) collected from students in response to the question: What is your definition of metacognition?}

the monitoring of ones thing of critical thinking criteria as one is acquiring and assessing new information :)

i've not heard of it but dictionary defines- awareness and undertanding one's own thought processes. So i'm d ucing that it is knowing about how you think?

metacognition is where the learner takes active control of their learning+they think about how they are thinking

metacognition is the process where the student takes conscious control of the learning. The learner thinks about how they are thinking in a cognitive sense.

Metacognition is an awareness and understanding of how you think.

the process where a student takes conscious control of their learning. And thinks about how they are thinking in a cognitive sense.

awarenes + understandin of $1 \mathrm{~s}$ own $4 \mathrm{t}$ proceses esp regard as havin a role in directin those processes.

my definition of effective learning is evaluating my thinking so that my thoughts are as effective as possible and well organis

the study me memory-monitoring and self regulation

it is when the student takes control of the learning process $\mathrm{n}$ does it on their own

metacognition refers to thinking cant cognition, or to reason about the way we think

Being able to consciously control planning and selecting stratagies, and to adapt these factors when necessary, to make learning more effective.

awareness and understanding one's thinking and cognitive processes. thinking about thinking metacognition- awareness and understanding of ones own thought processes, esp. Regard as having a role in directing those processes.

Metacognition seems to be th process of thinking about thinking.

metacognition is self fe back on assessing the outcome and realising if changes are ne to be made to the process which produces them.

If sum1 has good Metacognition then they hav a good awareness \& can understnd there cognitive processes. They basicaly hav the ability to think bout thinkin.

this is the process where th student takes conscious control of the learning. The learner think about how he is thinkin in a cognitive sense

metacognition is when an individual has knowl ge about own thinking and knows of the factors that influence their thinking.

knowl ge of your own thoughts and the factors that influence your thinking

being aware of and controling cognitive proces

metacognition means strengths of memory and behavior

An individuals knowl ge \& awareness concernin his/her own learnin \& thought processes.these processes include memory, intentional learnin \& recollection.

metacognition is an awareness of process of learning.consists of developin plan of action,maintainin monitorin of plan and evaluatin plan (self reflection)

metacognition is “thinking about thinking \& learning 2 learn"basically it's understanding how to approach things $\&$ how 2 fulfill a task all by planning ahead.

Metacognition is when a pupil has complete control and understanding of their learning.

metacognition is the recognition on the part of the learner that the learning is taking place, or has taken place

reflectin on ones on thoughts through continu learning and assessment. Being open to new information and being critically mind .

Metacognition is a conscious awareness of ones own learning and the processes involv in that learning

this means awareness and understanding one's thinking and cognitive processes, thinking about thinking. Have a nice day :-)

it is when a student is in control of their learning \& the processes involv . It refers to cognition, reasoning our own thinking, and critical analysis.

this means understandin \& bein aware of how u think, and then usin this to maximise learnin. An appreciation of what someone already knows and the skills and knowl ge that they may require to learn more efficiently and reliably. 
metacognition is the awareness and understanding ones thinking and cognitive processes ; thinking about thinking!

to think about ones thinking

it is argulation of your memory and to learn and evaluate your potential thinking awareness and understanding one's thinking and cognitive processes thinking about thinking metacognition is thinking about thinking, how u $\mathrm{r}$ going 2 remember things ur learning etc.

Itmeans taking into account other peoples learnings styles and processes and bein considerate so that all styles $r$ address 4the benefit of all individuals.

metacognition refers to an awareness of the process of learning, and our understanding of our own learning process.

metacognition is thinking about thinking and it affects study decisions.it involves conscious and unconscious knowl ge.

It means the understanding of the cognitive process, which involves self reflection, self responding $\mathrm{n}$ initiative as well as goal setting $\mathrm{n}$ time management.

the simplest xplanation i found was 'thinking about thinking'. Metacognition involves identifying, completing and evaluating a thinking pocess or task

this means ... Students consciously take control of their learning and are aware of their cognitive processes.

This means understanding about how people think metacognition is the recognition of a learners own learning style.

awareness $n$ understandin one's thinkin $n$ cognitive processes-thinkin about thinkin. 